\title{
Results of modeling the behavior of the subgrade with pressure injection of solidifying solutions
}

\author{
Aleksey Lanis ${ }^{1, *}$ \\ ${ }^{1}$ Siberian Transport University, Dusi Kovalchuk st., 191, 630049, Novosibirsk, Russia
}

\begin{abstract}
Further development of railway transport is possible through increasing the traffic intensity by increasing the weight and number of trains and their speed. The growth of defects and deformations of the subgrade is inevitable, and the issues related to the reinforcement of soils of the subgrade are relevant. Measures that result in reliable operation of railway infrastructure facilities can be implemented during major repairs or new construction, which requires traffic restriction and leads to additional economic costs. Thus, one of the priority issues is the development and implementation of ways to reinforce the subgrade of railways without traffic restriction. Such methods can include pressure injection, as well as various modifications of this method based on combining known methods of reinforcement and injection.
\end{abstract}

\section{Introduction}

The method of pressure injection is one of the effective methods of reinforcing the subgrade and the foundations of railways and highways, carried out by injecting a binding solution into the soil under high pressure [1]. Its difference from other injection methods lies in the fact that the injection of the solidifying solution is carried out breaking the soil structure, while it spreads through the resulting cracks and weak interlayers in the massif. As a result, a rigid framework is formed from the solidified solution and soil is compacted in the areas of its compression. Compacting of soil by injection of solidifying solutions has a favorable effect of a significant reduction in the filtration coefficient, which can be used to create the cut-off walls.

For the effective design of measures to strengthen the soil of the subgrade, it is necessary not only to specify the cause of defects and deformations, but also to identify the places of their formation, the weakened zones [2]. The distribution of the injected solution firstly occurs over these weakened zones. Long-term studies have shown that the mechanical characteristics of soil reinforced by the method of pressure injection depend first of all on the volume and properties of the solidified solution. The properties of the injectable solution are selected by the ratio of binder and aggregate. This issue is widely considered in the literature and does not require, in the author's opinion, additional research. The most interesting is the stress-strain state of the soil before reinforcement and around

\footnotetext{
*Corresponding author: lanisal@stu.ru
} 
various inclusions from the solidified solution after reinforcement, the direction of its distribution. The emergence of defects and deformations in the subgrade, the formation of weak interlayers, the distribution of the injected solution, and other similar internal processes cannot be described by known mathematical methods. Despite many known theoretical descriptions of certain aspects of the behavior of bulk materials and soils, the geotechnical problems considered above are still not completely solved because of the increased complexity.

\section{Methods}

To solve these problems, the discrete element method is proposed on the basis of its modification [3]. In the three-dimensional case (3D), the studied region of the model medium in DEM consists of $N$ separate discrete elements of the spherical shape. Each $i$-th element has one geometric and five physical and mechanical parameters: $r_{i}, \rho_{i}, \varphi_{i}, E_{i}, C_{i}, E_{c i}$ - radius, density, angle of contact friction, modulus of elasticity, cohesion, and modulus of elasticity of the virtual clutch spring, respectively. Such a spring is an elastic element - a piece whose ends are rigidly attached to the surfaces of the contacting balls. At the initial time, the length of the virtual clutch spring is zero.

The absence of the Poisson's ratio in the determining parameters of the elements is explained by the fact that in the DEM, when tensile or compressive forces are applied to the model sample, its longitudinal (in the direction of the forces) and transverse deformations are realized automatically by the movement of discrete elements under the action of these forces. The changes in the shape of the discrete elements, which arise when they contact, are not included in the DEM, and the calculation of the contact forces includes the magnitude of their mutual overlap. Thus, in DEM, the deformation of the model medium means not deformation of the discrete elements, from which it is composed, but changing their position relative to each other.

The $i$-th and $j$-th discrete elements interact with each other on their contacts by means of repulsion $\vec{F}_{n i j}$, friction $\vec{F}_{i j}$, and cohesion $\vec{F}_{c i j}$ forces. For each $i$-th element, the equations of motion are:

$$
\begin{gathered}
m_{i} \frac{d^{2} \vec{r}_{n i}}{d t^{2}}=\sum_{j=1}^{n_{\tau}}\left(\vec{F}_{n i j}+\vec{F}_{i j}\right)+\sum_{j=1}^{n_{c}}\left(\vec{F}_{c i j}\right)+m_{i} \vec{g}, \\
I_{i} \frac{d^{2} \vec{\alpha}_{i}}{d t^{2}}=\sum_{j=1}^{n_{\tau}}\left(\vec{F}_{\tau i j} \times \vec{r}_{i i j}\right)+\sum_{j=1}^{n_{c}}\left(\vec{F}_{c i j} \times \vec{r}_{c i j}\right),
\end{gathered}
$$

and initial conditions when $t=0$

$$
\vec{r}_{n i}=\vec{r}_{n i 0}, \quad \vec{\alpha}_{i}=\vec{\alpha}_{i 0}, \quad \vec{v}_{i}=\vec{v}_{i 0}, \quad \vec{\omega}_{i}=\vec{\omega}_{i 0},
$$

where $i=1, \ldots, N ; n_{\tau}$ - the number of elements contacting the $i$-th element by means of forces $\vec{F}_{n i j}$ and $\vec{F}_{\tau i j} ; n_{c}$ - the number of elements contacting the $i$-th element by means of forces $\vec{F}_{c i j} ; m_{i}, I_{i}, \vec{r}_{n i}, \vec{\alpha}_{i}, \vec{v}_{i}, \vec{\omega}_{i}$ - mass, moment of inertia, position vector for the center of mass, angle of rotation, velocity of the center, angular velocity of the $i$-th element; $\vec{g}$ - 
acceleration of gravity; $\vec{r}_{i j}, \vec{r}_{c i j}$ - position vectors drawn from the center of the $i$-th element to the points of application of forces $\vec{F}_{i i j}, \vec{F}_{c i j}$, respectively.

The repulsive force $\vec{F}_{n i j}$ acting from the side of $j$-th to the $i$-th element arises when they are overlapped, i.e. when $\delta_{n i j}>0$, where $\delta_{n i j}=r_{i}+r_{j}-l_{i j}$ - the magnitude of overlapping, $l_{i j}$ the distance between the centers of the balls. The force $\vec{F}_{n i j}$ is directed along the line passing through the centers of the elements and in absolute value is assumed to be equal to:

$$
F_{n i j}=\frac{2 E_{i} E_{j}}{E_{i}+E_{j}} \min \left(r_{i}, r_{j}\right) \delta_{n i j}
$$

The friction force $\vec{F}_{i j}$ is directed against the motion of the $i$-th element relative to the $j$ th one and is assumed to be equal to:

$$
\vec{F}_{i i j}=-\frac{\vec{v}_{i i j}}{\left|\vec{v}_{i i j}\right|} \min \left(\operatorname{tg} \varphi_{i}, \operatorname{tg} \varphi_{j}\right) F_{n i j},
$$

where $\vec{v}_{i j}$-tangential component of the velocity of the $i$-th element relative to the $j$-th one at the point of their contact. We believe that the friction at the contacts of the elements is effective after disrupting the cohesion between them.

In the process of translational and rotational movements of balls, the ends of the virtual clutch spring between the $i$-th and $j$-th elements diverge; a cohesive force $\vec{F}_{c i j}$ occurs between them, directed along the spring and in absolute value is equal to:

$$
F_{c i j}=\left\{\begin{array}{l}
2 \min \left(r_{i}, r_{j}\right) E_{c i j} \delta_{c i j}, \quad \delta_{c i j}<\delta_{c i j}^{*}, \\
0, \quad \delta_{c i j} \geq \delta_{c i j}^{*}
\end{array}\right.
$$

where $\delta_{c i j}$ - the length of the virtual clutch spring, $\delta_{c i j}^{*}=2 \min \left(r_{i}, r_{j}\right) \frac{C_{i j}}{E_{c i j}}, C_{i j}=\min \left(C_{i}, C_{j}\right)$, $E_{c i j}=\frac{2 E_{c i} E_{c j}}{E_{c i}+E_{c j}}$. If $\delta_{c i j} \geq \delta_{c i j}^{*}$, then the cohesion is disrupted. It should be noted that in calculations, instead of $E_{c i j}$, it is more convenient to use the parameter $\varepsilon_{c i j}^{*}=\frac{C_{i j}}{E_{c i j}}$. In this case, $\delta_{c i j}^{*}=2 \min \left(r_{i}, r_{j}\right) \varepsilon_{c i j}^{*}$.

In the two-dimensional case (2D), cylindrical elements of unit height are used instead of balls, located parallel to each other and contacting each other along the generators. Twodimensional formulation simulates situations in which the subgrade loses stability. In this case, the loss of stability of the subgrade is understood as the collapse of the massif in the vicinity of slopes and the formation of cracks on its surface.

Fig. 1 shows the design area, which is the embankment formed from discrete elements with the following fixed characteristics: $r_{i}=0.04 \div 0.05 \mathrm{~m}, \rho_{i}=\rho=2500 \mathrm{~kg} / \mathrm{m}^{3}, \varphi_{i}=\varphi=17$ 
${ }^{\circ}, E_{i}=E=1000 \mathrm{MPa}, \varepsilon_{c i j}^{*}=\varepsilon_{c}^{*}=0.15, i=1, \ldots, \mathrm{N}$. The cohesion $C_{i}=C$ is a variable and its value is specified in the solution of the problem. Geometric parameters of the embankment $a$ and $\alpha$ are fixed and equal to $8 \mathrm{~m}$ and $38^{\circ}$, respectively, the value of its height $h$, as well as the value of cohesion $C$, is concretized when solving the problem.

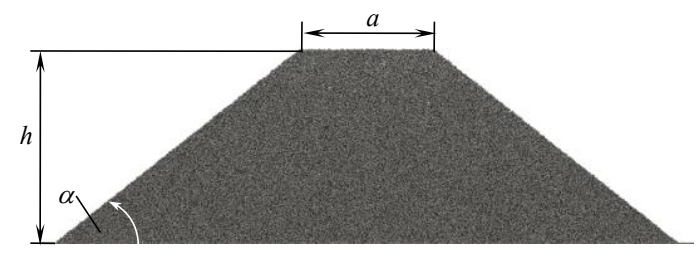

Fig. 1. The scheme of a bulk soil massif.

\section{Results}

Computer simulation of the loss of stability of the soil embankment is carried out as follows. First, the value of cohesion $C$ is selected, at which the embankment remains stable. Then, by successively decreasing the value of $C$, the situation is reached, when in some parts of the bulk massif cracks and/or slip lines develop. The value of $C$ obtained is assumed to be critical, at which the loss of stability of the embankment begins.

Numerical experiments show that as the height $h$ of the embankment increases, the value of cohesion $C$, at which the loss of its stability begins, grows. Fig. 2 shows the stages $t_{0}, t_{1}, t_{2}$ of the development of deformations in the soil embankment of different heights. The embankment with a height of $6 \mathrm{~m}$ begins to lose stability at $C=20 \mathrm{kPa}$, with a height of $9 \mathrm{~m}$ - at $C=25 \mathrm{kPa}$, and with a height of $12 \mathrm{~m}$ - at $C=30 \mathrm{kPa}$. In this case, the nature of the destruction and the sequence of its manifestation on the surface and inside the bulk massif for the three situations under consideration are different.

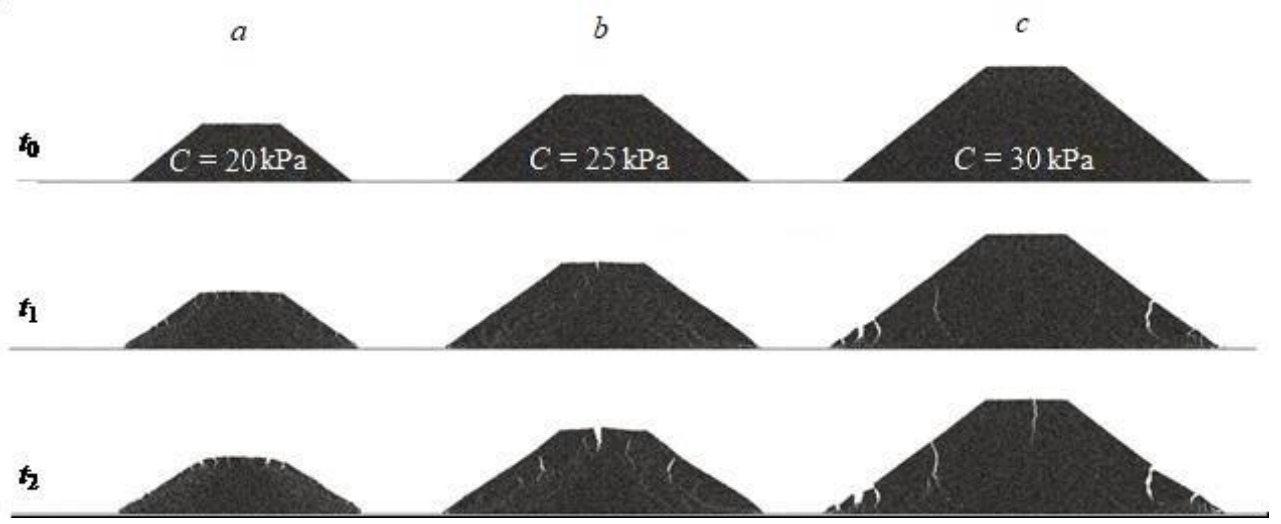

Fig. 2. The development of deformations in a soil embankment with a height of $6 \mathrm{~m}$ (a), $9 \mathrm{~m}$ (b) and $12 \mathrm{~m}(\mathrm{c})$.

In the first case (see Fig. 2a), cracks appear almost simultaneously at the horizontal surface of the embankment and on its slopes (stage $t_{1}$ ). Then, in the process of further deformation of the massif, cracks on the upper part of its surface are unfolded, and on the slopes are mainly healed (stage $t_{2}$ ). In the second case (see Fig. $2 b$ ), at first, nearly in the middle of the horizontal surface, the first crack appears (stage $t_{1}$ ). Further, this crack continues to unfold, and several slip lines appear inside the massif (stage $t_{2}$ ). In the third 
case (see Figure $2 c$ ), the first cracks appear on the slopes of the embankment closer to its base (stage $t_{1}$ ). With further deformation of the massif (stage $t_{2}$ ), a crack appears near the middle of the upper horizontal part of its surface, which is similar to one shown in Fig. $2 b$.

It is greatly important to check the adequacy (reliability) of the results obtained. The results of the performed calculations by the discrete element method can be compared with the results of modeling the stability of embankment slopes, performed using centrifugal modeling under the guidance of Professor T.G. Yakovleva in Moscow State University of Railway Engineering [4].

Fig. 3 shows the comparison of the results obtained in the experiment and in computer simulation. Fig. $3 a$ shows a view of the physical embankment with the slope angle of $\alpha=$ $38^{\circ}$ above the horizon, simulating a natural embankment with a height $h=9 \mathrm{~m}$, made of a light silt loam at $C=20 \mathrm{kPa}$. The experiment was carried out by the method of centrifugal modeling [4]. Violation of the limit equilibrium of the physical model, which has only been subjected to the action of body forces on centrifuge, begins with the formation of a crack almost strictly along the longitudinal axis of the model. A numerical experiment (see Fig. $3 b$ ) was carried out at $C=25 \mathrm{kPa}, h=9 \mathrm{~m}$. In computer simulation, as well as in the case of physical centrifugal modeling, a crack forms at the center of the embankment surface. In addition to it, several cracks and slip lines also developed inside the massif.

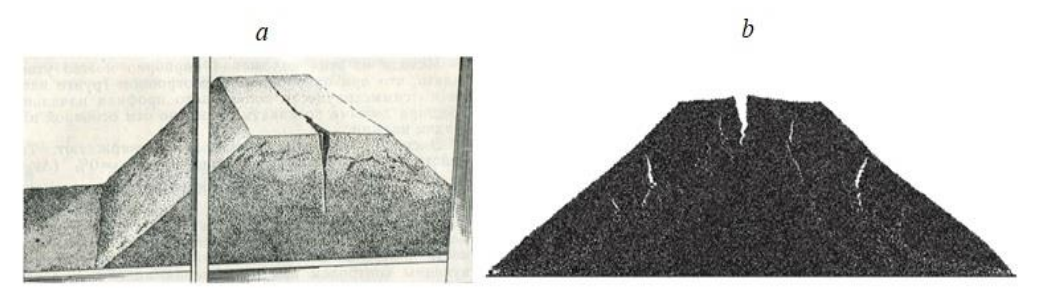

Fig. 3. Formation of a crack almost strictly along the longitudinal axis of the embankment model in the experiment (a) and in calculation (b).

a)

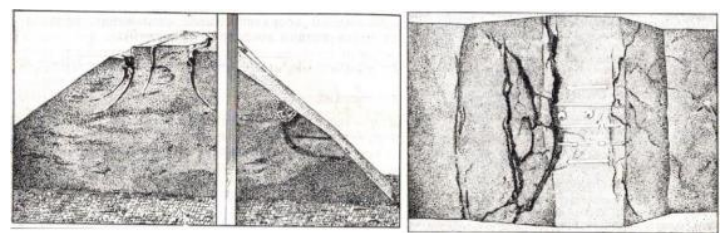

b)

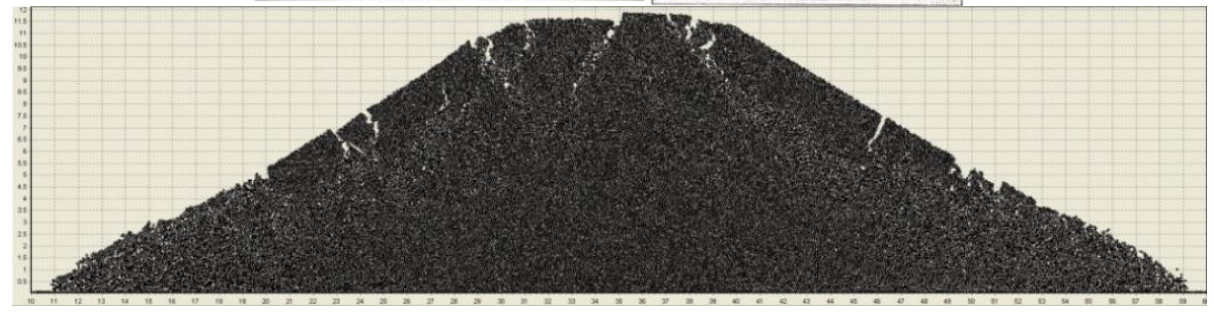

Fig. 4. Formation of cracks on the embankment model with a height of $6 \mathrm{~m}$ in the experiment (a) and in calculation (b).

Computer modeling has shown that the nature of the destruction and the sequence of its manifestation on the surface and inside the bulk massif highly depend on its height. In this case, the dependence of the limiting value of cohesion, at which the loss of stability of the embankment begins, is approximately linear on the range of the values of its heights from 6 to $12 \mathrm{~m}$ considered in this work. Comparison of the results of the experiment carried out by the method of centrifugal modeling with numerical results showed their close convergence. This is in favor of the fact that using the discrete element method, it is possible to 
successfully solve the problems of soil mechanics associated with railway track calculations. On real objects, these deformations are not observed externally, since the top of the subgrade is represented by the bulk material of the ballast prism, and the slopes are covered with ballast trails, vegetation. All these processes occur inside the subgrade, and their study is essential for the design of measures to prevent deformation. At the same time, numerous facts of the formation of ballast pockets and deformations on slopes are known. These are the external manifestations of internal processes revealed in the study.

The next problem solved in this paper is related to processes occurring in the subgrade when injecting solidifying solutions. Figure 5 shows the embankment, which has the form of a trapezoid, located in the plane $O x z$. Its height $h$ and the bases $l_{0}, l_{1}$ are 6,22 and $4 \mathrm{~m}$, respectively. It is formed from discrete elements $\Omega_{i}, i=1,2, \ldots, N$, where $N$ is a number equal to about 300,000 . They represent disks of unit thickness whose axes are perpendicular to the plane $O x z$, their centers are located in the plane $O x z$. The radius of disks located in the layer of the embankment at a level from zero to two meters is evenly distributed in the interval from 4 to $5 \mathrm{~mm}$, in the overlying layer in the interval from 2 to $2.5 \mathrm{~mm}$. The discs have a density of $\rho_{i}=2500 \mathrm{~kg} / \mathrm{m}^{3}$, the modulus of elasticity $E_{i}=1 \mathrm{GPa}$. The properties of the contacts between the disks are the following: sliding friction factor $k=0.4$, cohesion $C$ $=12 \mathrm{kPa}$, deformation of destruction of the cohesion element $\varepsilon^{*}=0.05$. Gravity is directed against the $O z$ axis. The disks forming the bottom base of the embankment are considered boundary discrete elements (BDE). We denote them by the symbol $B_{k}\left(k=k_{1}, k_{2}, \ldots, k_{n}, n\right.$ is the number of the BDE). The centers of $B_{k}$ are located on the $O x$ axis. The boundary conditions are superimposed on the elements of $B_{k}$ :

$$
\boldsymbol{v}_{k}=(0,0), \quad \omega_{k}=0,
$$

where $\boldsymbol{v}_{k}$ - the velocity vector of the center of the $k$-th element; $\omega_{k}$ - the angular velocity.

$t_{o}$
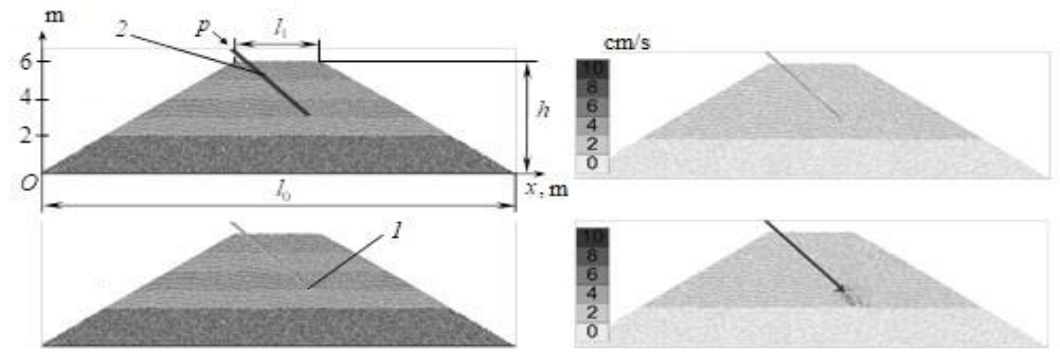

$t_{2}$
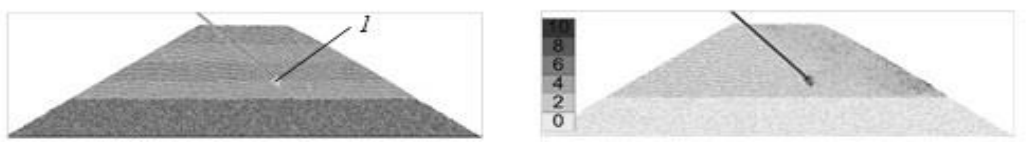

$t_{3}$
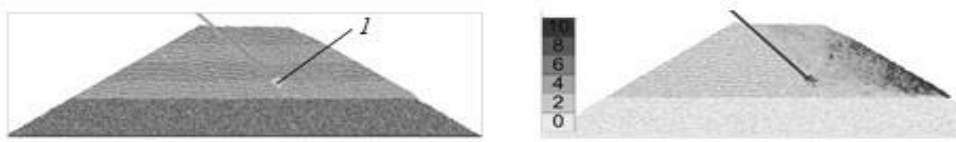

$t_{4}$

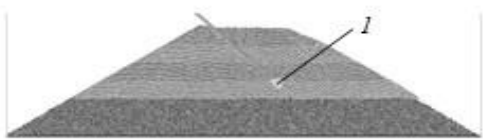

a)

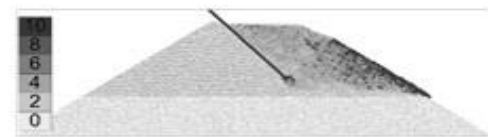

b)

Fig. 5. Stages $t_{0}-t_{4}$ of injecting the solution 1 under the pressure $p$ through a pipe 2 into a bulk massif with the characteristics $\rho=1800 \mathrm{~kg} / \mathrm{m}^{3} ; \varphi=0.3 ; c=12 \mathrm{kPa}: a$ - deformed state; $b$ - velocity field. 
The walls of the hollow pipe are formed from discrete elements with a radius of $1 \mathrm{~cm}$, the centers of which are located on parallel segments with length of $5 \mathrm{~m}$, located at a distance of $5 \mathrm{~cm}$ from each other. These elements penetrate at an angle of $45^{\circ}$ to the upper base of the embankment to a depth of $3 \mathrm{~m}$ (see Fig. 6). Then, the boundary conditions are superimposed on them (3). Further, elements are injected in the pipe under the pressure $p$ in the form of the same discs with a radius of $1 \mathrm{~cm}$, which model the solution.

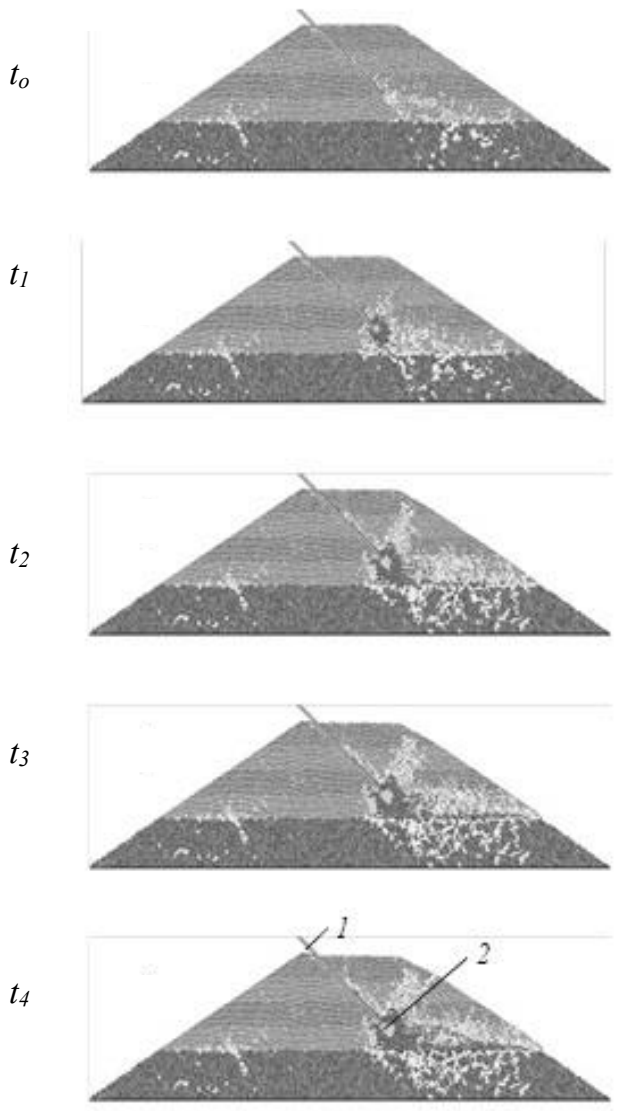

a)
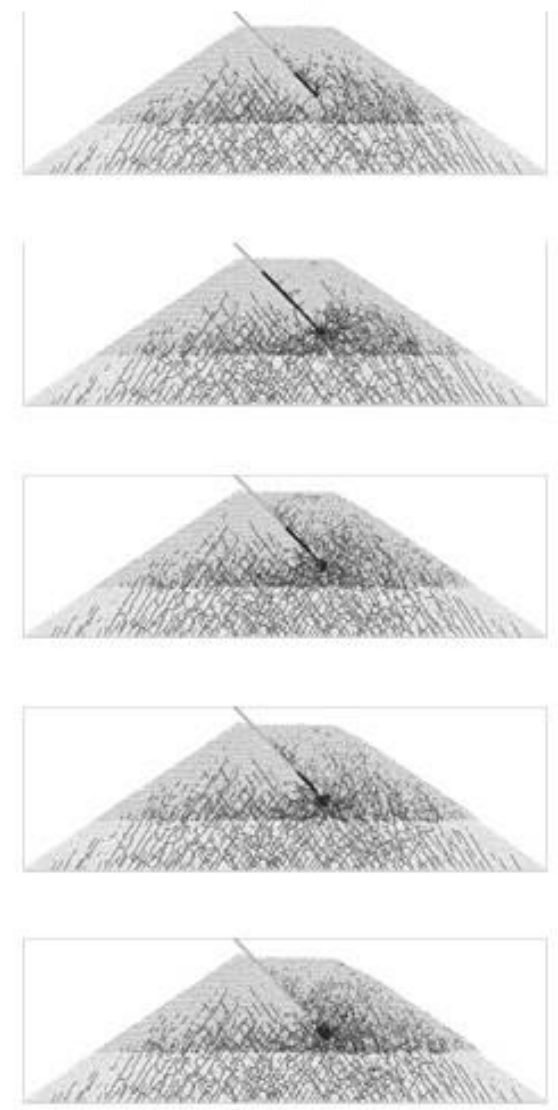

b)

Fig. 6. Stages t $0-t 4$ of injecting the solution 1 under the pressure $p$ through a pipe 2 into a bulk massif with the characteristics $\rho=1800 \mathrm{~kg} / \mathrm{m} 3 ; \mathrm{k}=0.3 ; \mathrm{c}=12 \mathrm{kPa}$ : a - rupture of deformations (yellow particles have at least one disruption of cohesion with neighboring particles; red particles - all cohesion contacts at the particle are destroyed); $b$ - fields of stresses normal to the contacts of particles (the pressure of the solution at the outlet from the pipe varies from 2 to $10 \mathrm{MPa}$ ).

Analyzing the figure 6, it can be concluded that, as a result of the probabilistic approach using the modified model of a soil embankment, it is possible to obtain directions for the formation of breaks in the soil structure of the subgrade and the injection pressure values, based on the discrete elements method.

According to the shape of disruptions, it is possible to track the formation of interlayers of the solidified solution. It is noted that for soil embankments, when injecting in a certain range of pressure values, the surface and slopes are raised, which is unacceptable for the operated railway subgrade. 


\section{Conclusion}

As a result of the studies conducted on modeling the behavior of the subgrade, results were obtained for determining the formation sites of weakened zones, followed by the formation of defects and deformations in the embankment. It is noted that the revealed internal processes in the embankments for the subgrade manifests only in the course of time in the form of ballast pockets and deformations on the slopes. Modified model based on the discrete element method used in the study allows simulating the distribution of the injected solution in the subgrade. It is possible to obtain directions and optimum values of the pressure of the solution distribution.

\section{References}

1. A.L. Lanis, Izvestiya Transsiba 3(27), 117-124 (2016)

2. A. Lanis, D. Razuvaev, Sciences in Cold and Ar-id Regions 9(3), 205-212 (2017)

3. A.L. Lanis, G.N. Khan, Bulletin of TSUAB 1, 273-281 (2013)

4. T.G. Yakovleva, D.I. Ivanov, Simulation of strength and stability of the subgrade (Transport, Moscow, 1980) 